\title{
Evolution of nuclear dualism in ciliates: a reanalysis in light of recent molecular data
}

Department of Biological Sciences, Smith College, Northampton, MA 01063, and Program in Organismic and Evolutionary Biology, UMASS-Amherst, Amherst MA 01003, USA

\author{
Laura A. Katz \\ Tel: +1 413585 3825. Fax: + 1413585 3786. e-mail: LKatz@Smith.edu
}

Keywords: Ciliophora, genome evolution, chromosomal rearrangements, origin of macronucleus, epigenetic effects

\section{INTRODUCTION}

Ciliates are a diverse, monophyletic assemblage of microbial eukaryotes defined by the presence of cilia in at least one phase of their life cycle and by the division of nuclear function into two types of nuclei - the 'germline' micronucleus (MIC) and the 'somatic' macronucleus (MAC) (Hausmann \& Bradbury, 1996; Prescott, 1994; Raikov, 1996). During conjugation, meiotic products of the MIC are exchanged between cells and fuse to form a zygotic nucleus (Fig. 1). This zygotic nucleus then divides mitotically and daughter nuclei either differentiate into MACs or remain unprocessed as MICs. During cell division, which occurs asexually in ciliates, MICs divide by mitosis while the MACs of all but one class of ciliates divide by an unusual form of nuclear division termed 'amitosis'.

Abbreviations: IES, internally excised sequences; MAC, macronucleus; MIC, micronucleus; $\operatorname{Div}^{+}$MAC, macronucleus capable of dividing.
Neither condensed chromosomes nor mitotic spindles are seen during MAC division. Instead, chromatin moves in an atypical manner along microtubules located either within or outside the MAC (Raikov, 1996). MAC division is absent from the class Karyorelictea, where a new MAC must differentiate from the MIC following every cell division (Raikov, 1996).

The development of the MAC occurs through chromosomal rearrangements, including fragmentation, elimination of internal sequences and amplification of the processed chromosomes. To date, most studies of the molecular basis of nuclear dimorphism have focused on five genera representing two of the nine ciliate classes: Tetrahymena and Paramecium (class Oligohymenophorea) and Oxytricha, Euplotes and Stylonychia (class Spirotrichea). Relatively limited chromosomal processing marks the first two genera, while the genomes of spirotrichs are extensively fragmented to create MACs with hundreds to thousands of copies of 'gene-sized chromosomes' (defined here as 


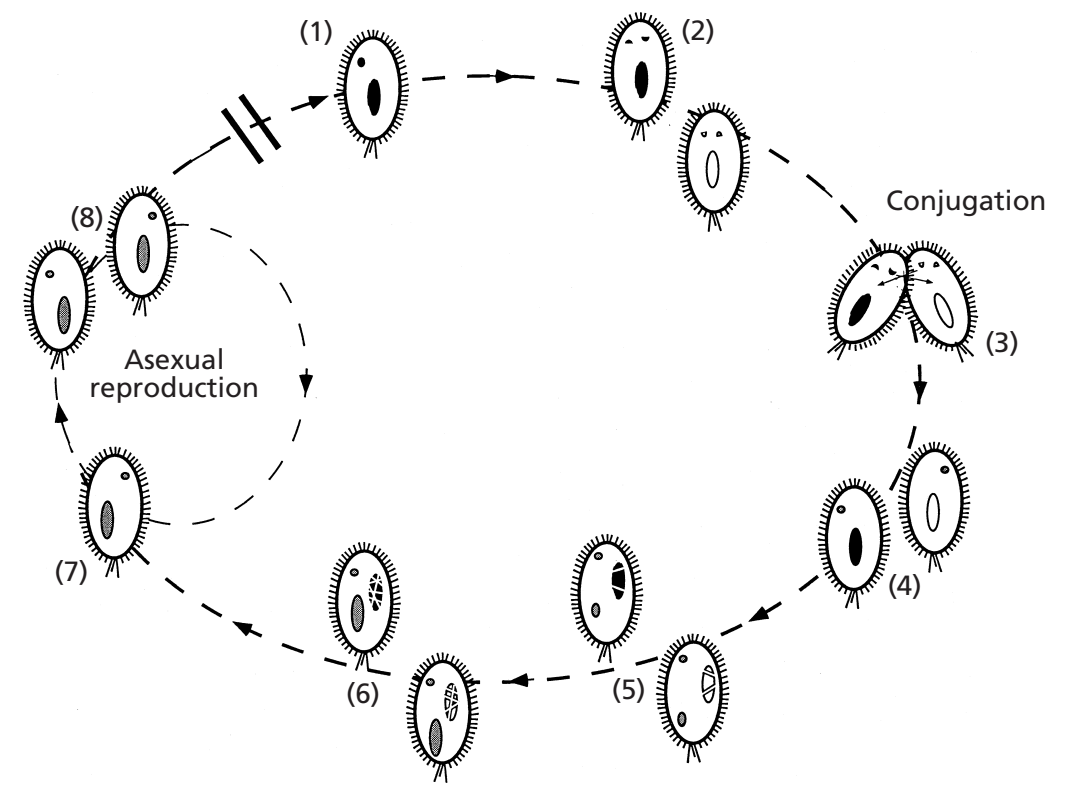

Fig. 1. Generalized ciliate life cycle. (1) A ciliate with its MIC (black circle) and MAC (black oval). (2) Two ciliates of different mating types preparing for conjugation - the MICs undergo meiosis. (3) Conjugation: haploid MICs are exchanged generating (4) ciliates with genetically novel zygotic nuclei (grey circles). (5) The zygotic nucleus divides and one daughter nucleus begins to differentiate into a MAC; the old MAC begins to fragment. (6) A new MAC has differentiated and the old MAC degrades. $(7,8)$ The ciliate divides asexually (e.g. Coyne et al., 1996; Prescott, 1994).

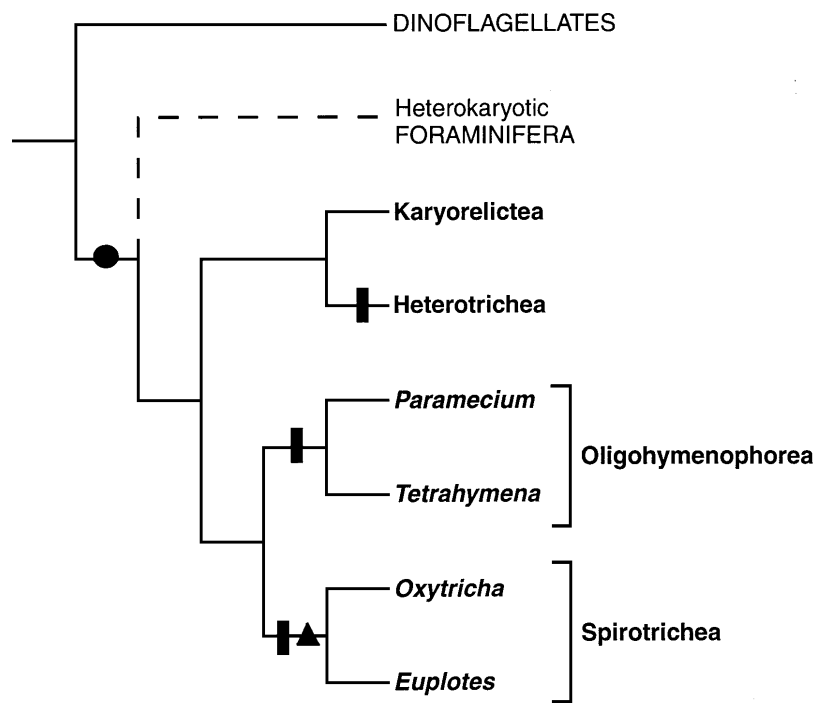

Fig. 2. The hypothesis of Orias $(1991 a, b)$ redrawn from Herrick (1994). Ciliates are shown in bold. The dashed line leading to foraminifera indicates the speculative nature of this portion of the topology. Black circles, MAC differentiation; black triangles, gene-size MAC 'chromosomes'; black rectangles, origin of $\mathrm{Div}^{+}$ MAC.

DNA molecules averaging $2-4 \mathrm{~kb}$ in length with telomeres on either end; Prescott, 1994: Coyne et al., 1996).

While common features of chromosomal rearrangements do exist in all ciliates, differences in MAC development and MAC structures among a limited sample of taxa led to the speculation that components of this system have evolved independently multiple times (Fig. 2; Herrick, 1994; Orias, 1991a). However, recent data indicating an even greater diversity among MACs, reviewed below, challenge the validity of this model. Combining these data with observations on a potential epigenetic effect during MAC development provides a new model that requires only a single origin of a relatively plastic mechanism of chromosomal rearrangements to explain the variation in MAC structure observed among ciliates.

\section{CURRENT HYPOTHESIS AND RECENT DATA}

\section{Macronuclear division}

The evolution of MACs capable of dividing ( $\mathrm{Div}^{+}$ MACs) remains unclear as there are two equally parsimonious ways to reconstruct the history of this characteristic of ciliates. As described above, Div ${ }^{+}$ MACs are found in all classes of ciliates except the Karyorelictea. Where $\mathrm{Div}^{+}$MACs occur, the unconventional process of amitosis is required to divide the polyploid, processed chromosomes of MACs. While mitosis in the micronucleus, the more 'typical' eukaryotic nucleus, occurs through intranuclear orthomitosis (spindle fibres arise at opposite poles within MICs; Raikov, 1982), $\mathrm{Div}^{+}$MACs divide by amitosis with microtubules either outside the MAC (in the class Heterotrichea) or inside the MAC (in all other ciliates, subphylum Intramacronucleata; Lynn, 1996).

According to one school of thought, $\mathrm{Div}^{-}$MACs represent the ancestral state for ciliates. Raikov (1976, 1982) argued that the nearly diploid, Div $^{-}$MACs of karyorelictids represent an ancestral state that developed early in the evolution of nuclear dimorphism of ciliates. Similarly, Orias reasoned that (a) nuclear dimorphism in ciliates is homologous to that of the heterokaryotic foraminifera, and (b) the inability of the somatic nucleus of both the foraminifera and the karyorelictids to divide is a shared ancestral character (Orias, 1991a, b). Orias (1991a, b) and later Herrick (1994; Fig. 2) further argued that the variability in 


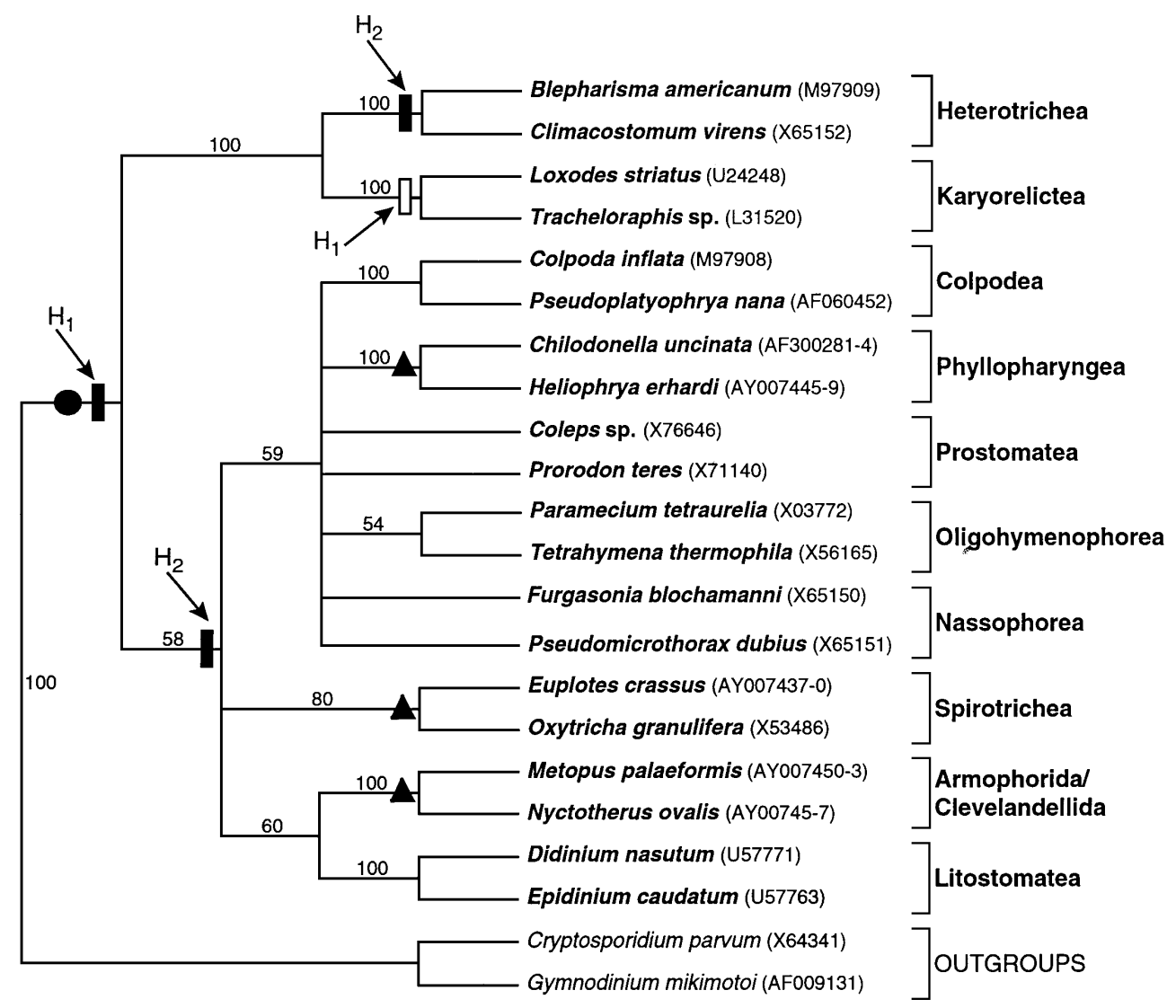

Fig. 3. Evolution of nuclear dualism in ciliates. Ciliates are shown in bold. Phylogeny based on bootstrap analysis of smallsubunit rRNA sequences in PAUP* 4.0b4a (Swofford, 1999) with likelihood parameters estimated by the hierarchical ratio tests in MODELTEST v. 3.0 (Posada \& Crandall, 1998). Sequences were aligned in CLUSTAL W (DNASTAR, Madison, WI, USA) with a gap penalty of 30 and a gap length penalty of 5 . Gaps and ambiguously aligned regions were removed for the analysis. The bootstrap values above the lines are based on 1000 replicates performed using a heuristic search. Sequences of Chilodonella uncinata and Heliophrya erhardi, Euplotes crassus, Metopus palaeformis and Nyctotherus ovalis represent a consensus of four to five clones. Outgroups are the dinoflagellate Gymnodinium mikimotoi and apicomplexan Cryptosporidium parvum. Black circles, parentally mediated MAC differentiation; black triangles, gene-size MAC 'chromosomes'; black rectangles, origin of $\mathrm{Div}^{+} \mathrm{MAC}$; white rectangles, loss of $\mathrm{Div}^{+} \mathrm{MAC}$; $\mathrm{H}_{1}$, hypothesis 1 : single origin followed by loss; $\mathrm{H}_{2}$, hypothesis 2 : two origins.

MAC structure indicates at least three independent origins of $\mathrm{Div}^{+}$Macs in ciliates: one for the relatively unprocessed oligohymenophoran MAC, one for the dramatically processed spirotrich MAC and a third for the heterotrichs.

Since the models put forth by Orias $(1991 \mathrm{a}, \mathrm{b})$ and elaborated by Herrick (1994), our understanding of the origin of dimorphic nuclei in ciliates has been altered by the taxonomic placement of foraminifera with respect to ciliates. Both recent analyses of ultrastructure (Taylor, 1999) and molecular data (Darling et al., 1996; Pawlowski, 1996) do not support the sister status of ciliates and foraminifera; instead, these analyses place foraminifera towards the base of the eukaryotic tree. Hence, the origin of heterokaryotic nuclei in ciliates appears to be independent of that in foraminifera.

Based on analyses of small-subunit rRNA genealogies, Hammerschmidt et al. (1996) discussed the two equally parsimonious mappings for $\mathrm{Div}^{+}$MACs: (1) an early origin of MAC $\mathrm{Div}^{+}$followed by a loss in the Karyorelictea or (2) two independent origins of $\mathrm{Div}^{+}$ MACs, one in the heterotrichs and one in all of the other ciliates (see Fig. 3). The authors favour the former hypothesis and argue that the extranuclear microtubules involved in amitosis in the Heterotrichea represents a derived state (Hammerschmidt et al., 1996). Implicit in this argument is the assumption that having intranuclear microtubules is the ancestral state of all nuclear division in ciliates as this placement of microtubules is present in ciliate MICs and the MACs of ciliates in the subphylum Intramacronucleata. However, it is still possible that $\mathrm{Div}^{+}$MACs evolved from $\mathrm{Div}^{-} \mathrm{MACs}$ twice, once with intranuclear microtubules and once with extranuclear microtubules.

\section{Chromosomal rearrangements}

As with MAC division, the apparent lack of conservation of sequences involved in chromosomal rearrangements within and between the oligohymeno- 
Table 1. Summary of MAC processing in four genera of ciliates

IES excision, fidelity of removal of IES (internally excised sequences); IES structure, nature of repeats at ends of IES; CB fidelity, location of CB (chromosome breakage) points; CB 'signal', nature of sequence at CB. Based on information given by Coyne et al. (1996) and Klobutcher \& Herrick (1997).

\begin{tabular}{|lllll|}
\hline Genus & IES excision & IES structure & CB fidelity & CB 'signal' \\
\hline Tetrahymena & Imprecise & Variable & Precise & Conserved sequence \\
Paramecium & Precise & TAs & Variable & Variable \\
Oxytricha & Precise & Variable & Variable & Variable \\
Euplotes & Precise & TAs and others & Precise & Conserved sequence \\
\hline
\end{tabular}

phorans and spirotrichs studied to date has led to the hypothesis of multiple independent origins of chromosomal rearrangements within ciliates (Table 1; Coyne et al., 1996; Klobutcher \& Herrick, 1997). As fragmentation is coupled to de novo synthesis of telomeres on MAC chromosomes (Coyne et al., 1996; Prescott, 1994), comparisons of the location of MAC telomeres with the corresponding sequences in the MIC allows identification of fragmentation sites (chromosomal breakage points) that are the site of telomere addition in MAC chromosomes. A conserved, polypurine rich chromosomal breakage sequence (cbs) has been identified in Tetrahymena thermophila (class Oligohymenophorea) and its close relatives (Coyne \& Yao, 1996; Yao et al., 1987). However, no evidence of a conserved sequence has been found in Paramecium (class Oligohymenophorea), where telomere addition can occur over a 200-800 bp region in developing MACs (Steele et al., 1994). Within the class Spirotrichea there is also variation in chromosomal breakage points. As with Paramecium, there is no evidence of a conserved sequence at chromosomal breakage points in Oxytricha (class Spirotrichea) where again there is some heterogeneity in the breakage sites (Herrick et al., 1987). In contrast, breakage is very precise in Euplotes crassus (class Spirotrichea) where a conserved $14 \mathrm{bp}$ sequence is associated with the telomere addition sites (Baird \& Klobutcher, 1989; Klobutcher et al., 1998). However, this sequence is not similar to the conserved cbs of Tetrahymena.

As with chromosomal breakage, analysis of the taxa sampled to date indicates that a diversity of mechanisms may be involved in the excision of internally excised sequences (IESs) within and between classes of ciliates. IESs are sequences of variable length always bounded by repeats that are removed during MAC development (Coyne et al., 1996; Klobutcher \& Herrick, 1997). Based on sequence comparisons, IESs can be classified either as transposable elements or as 'short' IESs (reviewed by Klobutcher \& Herrick, 1997). Several classes of transposable elements that are removed during macronuclear development have been found in the spirotrichs Oxytricha and Euplotes (Klobutcher \& Herrick, 1997). Transposable elements have not been found in either Paramecium or Tetrahymena (Klobutcher \& Herrick, 1997).
A second group of IESs, short non-transposable elements, has been found in all taxa examined to date, but vary in length and sequence both within and between classes (Klobutcher \& Herrick, 1997). 'Short' IESs are found within coding regions in both spirotrichs and Paramecium and are excised precisely. In Euplotes and Paramecium, 'short' IESs are flanked by 'TA' direct repeats and contain a conserved, eight nucleotide inverted repeat sequence (Klobutcher \& Herrick, 1995). (This same sequence is also found in the Tec transposons of E. crassus, leading to the hypothesis that these, and perhaps all, IESs are excised by a transposon-like mechanism; Klobutcher \& Herrick, 1997.) In contrast, the direct repeats flanking 'short' IESs in Oxytricha are variable between elements and no evidence of conserved sequences have been found to date (Ribas-Aparicio et al., 1987). The 'short' IESs in Tetrahymena are quite different as no IESs have been found interrupting coding sequences of $T$. thermophila and excision in this genus is imprecise (reviewed by Coyne et al., 1996). Also, although conserved sequences have been identified that are essential for excision of individual elements in Tetrahymena (Godiska \& Yao, 1990), no conserved features have been identified between IESs. The current interpretation for the lack of conserved features among ciliates is that the mechanisms underlying MAC differentiation, like the ability of MACs to divide, has arisen independently multiple times (Klobutcher \& Herrick, 1997).

\section{Macronuclear structure}

Another recent development in our understanding of the evolution of chromosomal rearrangements comes from data on the widespread distribution of extensive fragmentation. Extensive fragmentation has been best characterized from members of the classes Spirotrichea (e.g. Coyne et al., 1996; Klobutcher \& Herrick, 1997) and two closely related genera in the class Phyllopharyngea (Trithigmostoma and Chilodonella; Méténier \& Hufschmid, 1988). Recent data indicate that additional orders of phyllopharyngeans in the subclass Suctoria, as well as the genera Metopus and Nyctotherus, of disputed taxonomic position, also extensively fragment their genomes to create genesized chromosomes in their MACs (Riley \& Katz, 
2001). Southern hybridization of total DNA from several genera of phyllopharyngeans and from both Nyctotherus and Metopus reveal that the $\alpha$-tubulin gene of these taxa resides on 'chromosomes' less than $2 \mathrm{~kb}$ in length. According to the logic presented by Orias (1991a, b) and Herrick (1994), the non-sister status of the phyllopharyngeans, spirotrichs and disputed orders implies additional origins of $\mathrm{Div}^{+}$MACs in ciliates, one for each transition from less fragmented genomes to extensively fragmented genomes.

\section{A NEW MODEL}

By combining our knowledge of ciliate systematics with a growing understanding of MAC development, it is possible to construct a new model for the evolution of chromosomal processing in ciliates (Fig. 3). Under this model, a single origin of nuclear dimorphism in ciliates evolved along with a relatively plastic mechanism that has allowed for the evolution of diverse MACs. Until recently, the signals for chromosomal rearrangements were believed to be located predominantly in the zygotic nucleus, while the parental MAC, which eventually fragments and disappears, was thought to play a minimal role. Data on the role of the parental MAC in directing chromosomal rearrangements in the newly developing MACs of Paramecium and Tetrahymena (Duharcourt et al., 1995, 1998; Meyer et al., 1997; Meyer \& Duharcourt, 1996) provide a relatively plastic mechanism of chromosomal rearrangements that may have allowed for the observed diversity in chromosomal processing among ciliate MACs.

A growing number of experiments indicate that a trans-acting factor is specifically involved in regulating rearrangements during MAC development (Meyer \& Duharcourt, 1996). These experiments involve tracing the effects of microinjection of sequences into the parental MAC on the development of homologous sequences in the new MAC following conjugation. For example, injecting a high-copy number of surface A antigen genes into the parental MAC of Paramecium tetraurelia can rescue a mutant phenotype caused by the deletion of a portion of this gene from the parental MAC (Jessop-Murray et al., 1991). Injection of multiple copies of the surface $\mathrm{G}$ antigen gene (normally found at the end of a MAC chromosomes) into the parental MAC of Paramecium primaurelia can cause this segment of DNA to be deleted in the newly developing MAC (Meyer, 1992). Finally, injection of IESs into the parental MACs of both $P$. tetraurelia (Duharcourt et al., 1998) and T. thermophila (Chalker \& Yao, 1996) specifically prevents the excision of matching sequences from the developing MAC. Although the nature of the mechanisms underlying these epigenetic phenomena is still debated, the data are consistent with a trans-acting factor operating between the parental and newly developing MAC.

Under the proposed model, the evolution of nuclear dualism in ciliates was associated with, or evolved because of, the evolution of a mechanism whereby the parental MAC directly affects the development of a new MAC. Such an epigenetic mechanism may have allowed for the evolution of diverse MAC genome structure, such as the multiple origins of extensive fragmentation, observed in ciliates. Moreover, the presence of an epigenetic mechanism could also allow coevolution between template and target sequences to occur independently in multiple lineages. This might result in the observed variation in cis-acting regulatory sequences required for IES excision and chromosomal breakage in different ciliate lineages. The proposed model provides a more plausible explanation for the dramatic variation we observe among ciliate MACs. Instead of having to argue for independent origins of MAC division and extensive fragmentation and mechanisms of chromosomal rearrangements, we can now envision a single origin of a relatively plastic, epigenetic mechanism that allowed for the dramatic variation observed among MACs.

Clearly, more data, particularly on the nature and distribution of an epigenetic mechanism, are needed to test the proposed model. Do trans-acting factors from the parental MAC affect the newly developing MACs of all ciliate classes or just of the oligohymenophorans Paramecium and Tetrahymena? Development of microinjection systems in other ciliates will be necessary to answer this question. Even if the parental MAC turns out to have a regulatory role during MAC development in all ciliates, we need additional comparative data on the mechanisms of chromosomal processing (i.e. IES excision and chromosomal breakage sequences) to determine if the mechanisms are homologous, or have components that are homologous, among ciliate lineages. Finally, the number of origins of $\mathrm{Div}^{+} \mathrm{MACs}$ remains a mystery as we cannot distinguish between a single origin of $\mathrm{Div}^{+}$MACs in the ancestor of all extant ciliates followed by a loss in the karyorelictids from two independent origins of $\mathrm{Div}^{+}$MACs, one in the heterotrichs and one in the subphylum Intramacronucleata (Fig. 3).

\section{ACKNOWLEDGEMENTS}

Many thanks to Jennifer L. Riley, Tom Doak, Erica LasekNesselquist and Eduardo Orias for their insightful and stimulating discussions on the evolution of ciliates. This work is supported by A. F. Blakeslee Funds administered through the National Academy of Sciences.

\section{REFERENCES}

Baird, S. E. \& Klobutcher, L. A. (1989). Characterization of chromosome fragmentation in two protozoans and identification of a candidate fragmentation sequence in Euplotes crassus. Genes Dev 3, 585-597.

Chalker, D. L. \& Yao, M.-C. (1996). Non-Mendelian, heritable blocks to DNA rearrangement are induced by loading the somatic nucleus of Tetrahymena thermophila with germ linelimited DNA. Mol Cell Biol 16, 3658-3667.

Coyne, R. S. \& Yao, M.-C. (1996). Evolutionary conservation of 
sequences directing chromosome breakage and rDNA palindrome formation in tetrahymenine ciliates. Genetics 144, 1479-1487.

Coyne, R. S., Chalker, D. L. \& Yao, M.-C. (1996). Genome downsizing during ciliate development: nuclear division of labor through chromosome restructuring. Annu Rev Genet 30, $557-578$.

Darling, K. F., Kroon, D., Wade, C. M. \& Brown, A. J. L. (1996). Molecular phylogeny of the planktic foraminifera. J Foraminifer Res 26, 324-330.

Duharcourt, S., Bulter, A. \& Meyer, E. (1995). Epigenetic selfregulation of developmental excision of an internal eliminated sequence in Paramecium tetraurelia. Genes Dev 9, 2065-2077.

Duharcourt, S., Keller, A. M. \& Meyer, E. (1998). Homologydependent maternal inhibition of developmental excision of internal eliminated sequences in Paramecium tetraurelia. Mol Cell Biol 18, 7075-7085.

Godiska, R. \& Yao, M. C. (1990). A programmed site-specific DNA rearrangement in Tetrahymena thermophila requires flanking polypurine tracts. Cell 61, 1237-1246.

Hammerschmidt, B., Schlegel, M., Lynn, D., Leipe, D. D., Sogin, M. L. \& Raikov, I. B. (1996). Insights into the evolution of nuclear dualism in the ciliates revealed by phylogenetic analysis of rRNA sequences. $J$ Eukaryot Microbiol 43, 225-230.

Hausmann, K. \& Bradbury, P. C. (1996). Ciliates: Cells as Organisms. Stuttgart: Gustav Fischer.

Herrick, G. (1994). Germline-soma relationships in ciliate protozoa: the inception and evolution of nuclear dimorphism in onecelled animals. Semin Dev Biol 5, 3-12.

Herrick, G., Hunter, D., Williams, K. \& Kotter, K. (1987). Alternative processing during development of a macronuclear chromosome family in Oxytricha fallax. Genes Dev 1, $1047-1058$

Jessop-Murray, H., Martin, L. D., Gilley, D., Preer, J. R., Jr \& Polisky, B. (1991). Permanent rescue of a non-Mendelian mutation of Paramecium by microinjection of specific DNA sequences. Genetics 129, 727-734.

Klobutcher, L. A. \& Herrick, G. (1995). Consensus inverted terminal repeat sequence of Paramecium IESs: resemblance to termini of Tc1-related and Euplotes Tec transposons. Nucleic Acids Res 23, 2006-2013.

Klobutcher, L. A. \& Herrick, G. (1997). Developmental genome reorganization in ciliated protozoa: the transposon link. Prog Nucleic Acid Res Mol Biol 56, 1-62.

Klobutcher, L. A., Gygax, S. E., Podoloff, J. D., Vermeesch, J. R., Price, C. M., Tebeau, C. M. \& Jahn, C. L. (1998). Conserved DNA sequences adjacent to chromosome fragmentation and telomere addition sites in Euplotes crassus. Nucleic Acids Res 26, $4230-4240$.

Lynn, D. H. (1996). My journey into ciliate systematics. $J$ Eukaryot Microbiol 43, 253-260.
Méténier, G. \& Hufschmid, J. D. (1988). Evidence of extensive fragmentation of macronuclear DNA in two non-hypotrichous ciliates. J Protozool 35, 71-73.

Meyer, E. (1992). Induction of specific macronuclear developmental mutations by microinjection of a cloned telomeric gene in Paramecium primaurelia. Genes Dev 6, 211-222.

Meyer, E. \& Duharcourt, S. (1996). Epigenetic programming of developmental genome rearrangements in ciliates. Cell 87, 9-12.

Meyer, E., Butler, A., Dubrana, K., Duharcourt, S. \& Caron, F. (1997). Sequence-specific epigenetic effects of the maternal somatic genome on developmental rearrangements of the zygotic genome in Paramecium primaurelia. Mol Cell Biol 17, 3589-3599.

Orias, E. (1991a). Evolution of amitosis of the ciliate macronucleus: gain of the capacity to divide. J Protozool 38, 217-221.

Orias, E. (1991b). On the evolution of the karyorelict ciliate life cycle: heterophasic ciliates and the origin of ciliate binary fission. Biosystems 25, 67-73.

Pawlowski, J., Bolivar, I., Fahrni, J. F., Cavalier Smith, T. \& Gouy, M. (1996). Early origin of foraminifera suggested by SSU rRNA gene sequences. Mol Biol Evol 13, 445-450.

Posada, D. \& Crandall, K. (1998). MODELTEST: testing the model of DNA substitution. Bioinformatics 14, 817-818.

Prescott, D. M. (1994). The DNA of ciliated protozoa. Microbiol Rev 58, 233-267.

Raikov, I. B. (1976). Evolution of macronuclear organization. Annu Rev Genet 10, 413-440.

Raikov, I. B. (1982). The Protozoan Nucleus: Morphology and Evolution. Wien: Springer.

Raikov, I. B. (1996). Nuclei of ciliates. In Ciliates: Cells as Organisms, pp. 221-242. Edited by K. Hausmann \& P. C. Bradbury. Stuttgart: Gustav Fischer.

Ribas-Aparicio, R. M., Sparkowski, J. J., Proulx, A. E., Mitchell, J. D. \& Klobutcher, L. A. (1987). Nucleic acid splicing events occur frequently during macronuclear development in the protozoan Oxytricha nova and involve the elimination of unique DNA. Genes Dev 1, 323-336.

Riley, J. L. \& Katz, L. A. (2001). Widespread distribution of extensive genome fragmentation in ciliates Mol Biol Evol 18, $1372-1377$

Steele, J. C., Barkocy-Gallagher, G. A., Preer, L. B. \& Preer, J. R. (1994). Developmentally excised sequences in micronuclear DNA of Paramecium. Proc Natl Acad Sci USA 91, 2255-2259.

Swofford, D. (1999). PAUP*: phylogenetic analysis using parsimony (*and other methods). Sinauer Associates, Sunderland, MA, USA.

Taylor, F. J. R. (1999). Ultrastructure as a control for protistan phylogeny. Am Nat 154, S125-S136.

Yao, M.-C., Zheng, K. \& Yao, C. H. (1987). A conserved nucleotide sequence at the site of developmentally regulated chromosomal breakage in Tetrahymena. Cell 5, 1260-1267. 